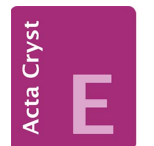

OPEN $\curvearrowright$ ACCESS COMMUNICATIONS

ISSN 2056-9890

\section{Crystal structure of 2,5-bis(diphenyl- phosphanyl)furan}

\section{Carla Martínez de León, Hugo Tlahuext and Jean-Michel Grévy*}

Centro de Investigaciones Químicas, Universidad Autónoma del Estado de Morelos, Av. Universidad 1001 Col. Chamilpa, CP 62209, Cuernavaca Mor., Mexico.

*Correspondence e-mail: jeanmichelg@gmail.com

Received 18 October 2015; accepted 4 November 2015

Edited by H. Stoeckli-Evans, University of Neuchâtel, Switzerland

In the title compound, $\mathrm{C}_{28} \mathrm{H}_{22} \mathrm{OP}_{2}$, each of the $\mathrm{P}$ atoms has an almost perfect pyramidal geometry, with $\mathrm{C}-\mathrm{P}-\mathrm{C}$ angles varying from $100.63(10)$ to $102.65(9)^{\circ}$. In the crystal, neighbouring molecules are linked via weak $\mathrm{C}-\mathrm{H} \cdots \pi$ interactions, forming supramolecular chains along the $b$-axis direction.

Keywords: crystal structure; bis(diphenylphosphanyl)furan; metal complexes; diphosphine ligands for catalysis; $\mathrm{C}-\mathrm{H} \cdots \pi$ interactions..

CCDC reference: 1435225

\section{Related literature}

For the uses of rigid diphosphine compounds in the preparation of homo- or hetero-bimetallic complexes, which have high potential for specific applications in catalytic processes, see: Kaeser et al. (2013); Xu et al. (2014). For the structural characteristics of these ligands providing control over the distance separating the two metallic centers and consequently, over the properties of the corresponding complexes, see: Brown \& Lucy (1986). For the synthesis of bis(diphenylphosphanyl)furan, see: Brown \& Canning (1983). For the resulting bimetallic complexes with Rh and Ir, see: Brown et al. (1984). For $\mathrm{C}-\mathrm{H} \cdots \pi$ interactions, see: Munshi \& Guru Row (2005).

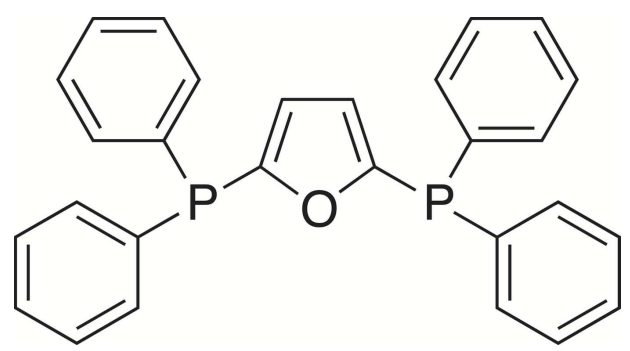

\section{Experimental}

\subsection{Crystal data}

$\mathrm{C}_{28} \mathrm{H}_{22} \mathrm{OP}_{2}$

$M_{r}=436.40$

Monoclinic, $P 2_{1} / c$

$a=10.7179(9) \AA$

$b=8.5559$ (7) $\AA$

$c=24.550(2) \AA$

$\beta=94.309(1)^{\circ}$

\subsection{Data collection}

Bruker SMART APEX CCD areadetector diffractometer

Absorption correction: multi-scan (SADABS; Bruker, 2000)

$T_{\min }=0.965, T_{\max }=0.975$

\subsection{Refinement}

$R\left[F^{2}>2 \sigma\left(F^{2}\right)\right]=0.046$

$w R\left(F^{2}\right)=0.106$

$S=1.17$

3952 reflections

$$
\begin{aligned}
& V=2244.9(3) \AA^{3} \\
& Z=4 \\
& \text { Mo } K \alpha \text { radiation } \\
& \mu=0.21 \mathrm{~mm}^{-1} \\
& T=100 \mathrm{~K} \\
& 0.17 \times 0.15 \times 0.12 \mathrm{~mm}
\end{aligned}
$$

17894 measured reflections 3952 independent reflections 3836 reflections with $I>2 \sigma(I)$ $R_{\text {int }}=0.045$
Table 1

Hydrogen-bond geometry $\left(\AA,^{\circ}\right)$.

$\mathrm{Cg}$ is the centroid of ring $\mathrm{C} 17-\mathrm{C} 22$.

\begin{tabular}{lllll}
\hline$D-\mathrm{H} \cdots A$ & $D-\mathrm{H}$ & $\mathrm{H} \cdots A$ & $D \cdots A$ & $D-\mathrm{H} \cdots A$ \\
\hline $\mathrm{C} 27-\mathrm{H} 27 \cdots C g^{\mathrm{i}}$ & 0.95 & 3.11 & $3.736(3)$ & 125 \\
\hline
\end{tabular}

Symmetry code: (i) $x, y-1, z$.

Data collection: SMART (Bruker, 2000); cell refinement: SAINT (Bruker, 2000); data reduction: $S A I N T$; $\operatorname{program}(\mathrm{s})$ used to solve structure: SHELXS97 (Sheldrick, 2008); program(s) used to refine structure: SHELXL97 (Sheldrick, 2008); molecular graphics: SHELXTL (Sheldrick, 2008); software used to prepare material for publication: SHELXTL, PLATON (Spek, 2009) and publCIF (Westrip, 2010).

\section{Acknowledgements}

This work was supported by CONACyT (project CB2009134528). CML is grateful for a scholarship (No. 276535) provided by this project.

Supporting information for this paper is available from the IUCr electronic archives (Reference: SU5229). 


\section{References}

Brown, J. M. \& Canning, L. R. (1983). J. Chem. Soc. Chem. Commun. pp. 460462.

Brown, J. M., Canning, L. R. \& Lucy, A. R. (1984). J. Chem. Soc. Chem. Commun. pp. 915-917.

Brown, J. M. \& Lucy, A. R. (1986). J. Organomet. Chem. 314, 241-246.

Bruker (2000). SMART, SAINT and SADABS. Bruker AXS Inc., Madison, Wisconsin, USA.
Kaeser, A., Mohankumar, M., Mohanraj, J., Monti, F., Holler, M., Cid, J.-J., Moudam, O., Nierengarten, I., Karmazin-Brelot, L., Duhayon, C., Delavaux-Nicot, B., Armaroli, N. \& Nierengarten, J.-F. (2013). Inorg. Chem. 52, 12140-12151.

Munshi, P. \& Guru Row, T. N. (2005). CrystEngComm, 7, 608-611.

Sheldrick, G. M. (2008). Acta Cryst. A64, 112-122.

Spek, A. L. (2009). Acta Cryst. D65, 148-155.

Westrip, S. P. (2010). J. Appl. Cryst. 43, 920-925.

Xu, K., Zheng, X., Wang, Z. \& Zhang, X. (2014). Chem. Eur. J. 20, 4357-4362. 


\section{supporting information}

Acta Cryst. (2015). E71, o922-o923 [https://doi.org/10.1107/S2056989015020964]

\section{Crystal structure of 2,5-bis(diphenylphosphanyl)furan}

\section{Carla Martínez de León, Hugo Tlahuext and Jean-Michel Grévy}

\section{S1. Commentary}

Rigid diphosphine compounds are important ligands for inorganic chemists as they can be used in the preparation of homo- or hetero-bimetallic complexes, which have high potential for specific applications in catalytic processes (Kaeser et al., 2013; Xu et al., 2014). The structural characteristics of these ligands provide control, among other things, over the distance separating the two metallic centers and consequently, over the properties of the corresponding complexes (Brown et al., 1986). Thus, as part of an investigation in the field, some thirty years ago (Brown et al., 1983) bis(diphenylphosphanyl)furan was synthesized for selective binuclear chelation and the resulting bimetallic complexes with $\mathrm{Rh}$ and Ir were isolated and latter tested in alkene hydrogenation (Brown et al., 1984), showing a poorer activity than the corresponding mononuclear analogues. However, we believe that this diphosphine ligand is still of great interest for an exhaustive coordination study. In former reports the ligand was not spectroscopically characterized, nor its crystal structure determined, so here we report its full characterization and solid-state structure studied by single-crystal X-ray diffraction.

The molecular structure of the title compound, Fig. 1, shows the two phosphorus atoms, P1 and P2, with almost perfect pyramidal geometry; the $\mathrm{C}-\mathrm{P}-\mathrm{C}$ angles are in a range of 100.63 (10) to 102.65 (9) ${ }^{\circ}$. The phenyl rings (C5-C10, $\mathrm{C} 11$ $-\mathrm{C} 16, \mathrm{C} 17-\mathrm{C} 22, \mathrm{C} 23-\mathrm{C} 28)$ and the furanyl ring $(\mathrm{C} 1-\mathrm{C} 4 / \mathrm{O} 1)$ are almost planar with r.m.s. deviations of 0.0024 , $0.0019,0.0026,0.0072$ and $0.0047 \AA$, respectively. The bond distances and angles have normal values.

In the crystal, the packing is stabilized via weak $\mathrm{C}-\mathrm{H} \cdots \pi$ interactions (Munshi \& Guru Row, 2005), involving adjacent molecules, forming a supramolecular chain along the $b$ axis direction (Table 1 and Fig. 2).

\section{S2. Synthesis and crystallization}

Although the title compound could be prepared in high yields by reaction between dilithiofuran and 2 equivalents of chlorodiphenylphosphine (Brown \& Canning, 1983), here it was obtained in 23\% yield as a side product from the synthesis of 2-(diphenylphosphanyl)furan: $\mathrm{nBuLi}$ in hexane solution $(8.25 \mathrm{mmol}$ ) was slowly added to a furane solution ( $8.25 \mathrm{mmol})$ in $15 \mathrm{ml}$ of $\mathrm{Et}_{2} \mathrm{O}$. After two hours of stirring at room temperature, a $15 \mathrm{ml}$ benzene solution of 1 equivalent of $\mathrm{Ph}_{2} \mathrm{PCl}$ was added drop wise at $273 \mathrm{~K}$. After stirring the mixture overnight, all volatiles were eliminated under reduced pressure, and the resulting oil was diluted in $\mathrm{CH}_{2} \mathrm{Cl}_{2}$ and then filtered over Celite. The pure diphosphine was obtained as the second product eluted on a silica column with the solvent system Hexane: $\mathrm{CH}_{2} \mathrm{Cl}_{2}$ (80:20). Yield: $23 \%$; m.p. $421 \mathrm{~K}$; $\mathrm{MS}(\mathrm{FAB}+) 436 \mathrm{~m} / \mathrm{z}(\mathrm{M}+) 40 \% ;{ }^{31} \mathrm{P}$ NMR $\left(\mathrm{CDCl}_{3}, 80 \mathrm{MHz}, 20^{\circ} \mathrm{C}\right)-27.4$ p.p.m; ${ }^{1} \mathrm{H}$ NMR $\left(400 \mathrm{MHz}, \mathrm{CDCl}_{3}, 20^{\circ} \mathrm{C}\right): \delta=$ $6.74(\mathrm{~m}, 2 \mathrm{H}), 7.23-7.36(\mathrm{~m}, 20 \mathrm{H}) ; \mathrm{RMN}^{13} \mathrm{C}\left(100 \mathrm{MHz}, \mathrm{CDCl}_{3}, 20^{\circ} \mathrm{C}\right) ; 122.62\left(\mathrm{dd}, 2 \mathrm{C},{ }^{2} J_{\mathrm{CP}}=27.8 \mathrm{~Hz},{ }^{3} J_{\mathrm{CP}}=7.3 \mathrm{~Hz}\right)$, $133.39\left(\mathrm{~d}, 8 \mathrm{C},{ }^{3} J_{\mathrm{CP}}=19 \mathrm{~Hz}\right), 128.3\left(\mathrm{~d}, 8 \mathrm{C},{ }^{3} J_{\mathrm{CP}}=7.3 \mathrm{~Hz}\right), 128.7(\mathrm{~s}, 4 \mathrm{C}), 157.8\left(\mathrm{~d}, 2 \mathrm{C},{ }^{1} J_{\mathrm{CP}}=24.9 \mathrm{~Hz}\right), 136.0\left(\mathrm{~d}, 4 \mathrm{C},{ }^{1} J_{\mathrm{CP}}=\right.$ $4.4 \mathrm{~Hz}$ ). Single crystals suitable for X-ray diffraction were grown by slow evaporation of a dichloromethane solution of the title compound at room temperature. 


\section{S3. Refinement}

Crystal data, data collection and structure refinement details are summarized in Table 2. $\mathrm{H}$ atoms were positioned geometrically and constrained using the riding-model approximation: $C-\mathrm{H}_{\text {phenyl }}=0.95 \AA$ with $U_{\text {iso }}\left(\mathrm{H}_{\text {phenyl }}\right)=1.2 U_{\text {eq }}(\mathrm{C})$, and $C$ - $\mathrm{H}_{\text {furanyl }}=0.95 \AA$, with $U_{\text {iso }}\left(\mathrm{H}_{\text {furanyl }}\right)=1.2 U_{\text {eq }}(\mathrm{C})$.

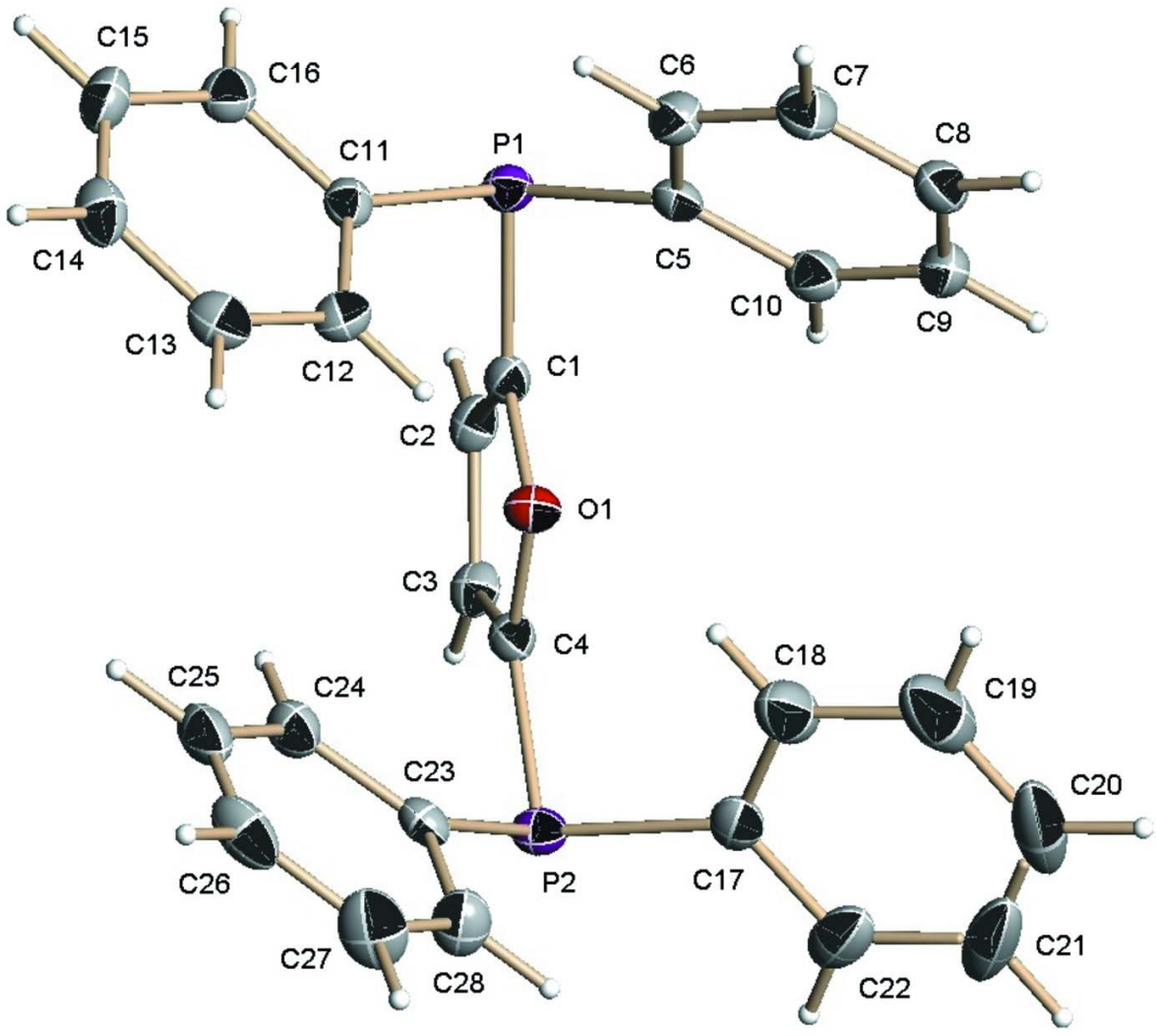

Figure 1

The molecular structure of the title compound, with atom labeling. Displacement ellipsoids are drawn at the 50\% probability level. 


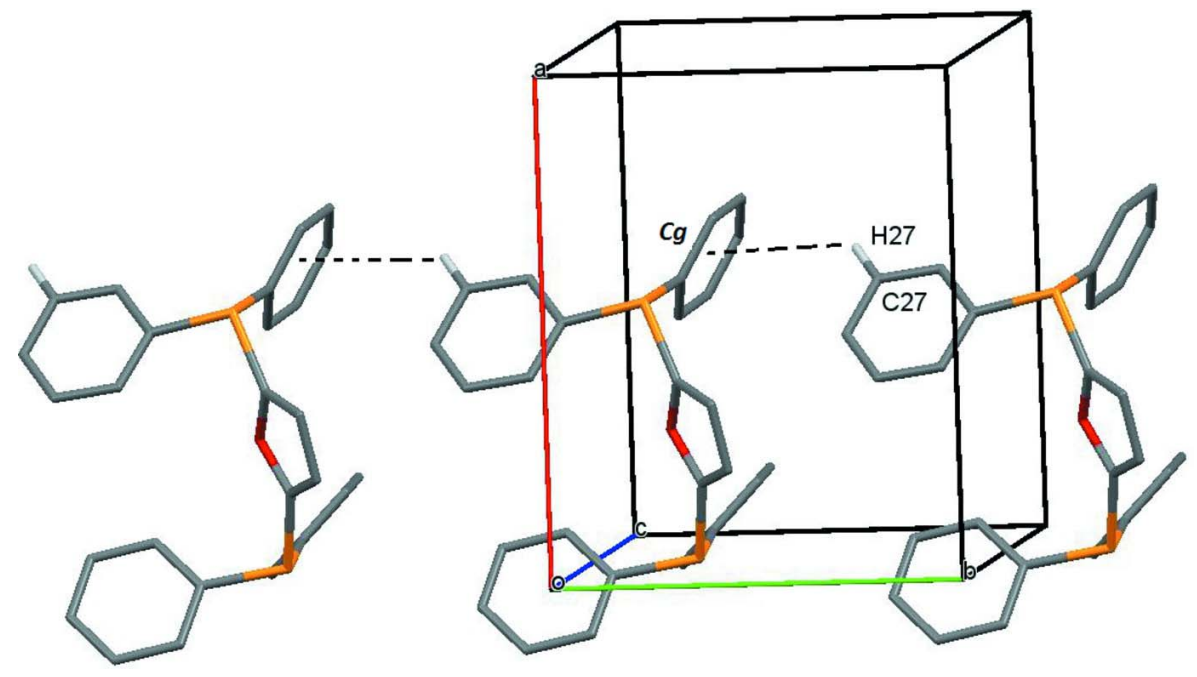

Figure 2

View of the $\mathrm{C}-\mathrm{H} \cdots \pi$ interactions (dashed lines; see Table 1) linking adjacent molecules. Hydrogen atoms not involved in these interactions have been omitted for clarity.

\section{2,5-Bis(diphenylphosphanyl)furan}

\section{Crystal data}

$\mathrm{C}_{28} \mathrm{H}_{22} \mathrm{OP}_{2}$

$M_{r}=436.40$

Monoclinic, $P 2_{1} / c$

Hall symbol: $-\mathrm{P} 2 \mathrm{ybc}$

$a=10.7179$ (9) $\AA$

$b=8.5559$ (7) $\AA$

$c=24.550(2) \AA$

$\beta=94.309(1)^{\circ}$

$V=2244.9(3) \AA^{3}$

$Z=4$

\section{Data collection}

Bruker SMART APEX CCD area-detector diffractometer

Radiation source: fine-focus sealed tube Graphite monochromator

Detector resolution: 8.3 pixels $\mathrm{mm}^{-1}$

phi and $\omega$ scans

Absorption correction: multi-scan

(SADABS; Bruker, 2000)

$T_{\text {min }}=0.965, T_{\max }=0.975$

\section{Refinement}

Refinement on $F^{2}$

Least-squares matrix: full

$R\left[F^{2}>2 \sigma\left(F^{2}\right)\right]=0.046$

$w R\left(F^{2}\right)=0.106$

$S=1.17$

3952 reflections

280 parameters

0 restraints
$F(000)=912$

$D_{\mathrm{x}}=1.291 \mathrm{Mg} \mathrm{m}^{-3}$

Mo $K \alpha$ radiation, $\lambda=0.71073 \AA$

Cell parameters from 7667 reflections

$\theta=2.4-28.3^{\circ}$

$\mu=0.21 \mathrm{~mm}^{-1}$

$T=100 \mathrm{~K}$

Block, colorless

$0.17 \times 0.15 \times 0.12 \mathrm{~mm}$

17894 measured reflections

3952 independent reflections

3836 reflections with $I>2 \sigma(I)$

$R_{\text {int }}=0.045$

$\theta_{\max }=25.0^{\circ}, \theta_{\min }=1.9^{\circ}$

$h=-12 \rightarrow 12$

$k=-9 \rightarrow 10$

$l=-29 \rightarrow 29$

Primary atom site location: structure-invariant direct methods

Secondary atom site location: difference Fourier map

Hydrogen site location: inferred from neighbouring sites

$\mathrm{H}$-atom parameters constrained 
$w=1 /\left[\sigma^{2}\left(F_{\mathrm{o}}^{2}\right)+(0.0276 P)^{2}+1.9895 P\right]$

where $P=\left(F_{\mathrm{o}}^{2}+2 F_{\mathrm{c}}^{2}\right) / 3$

$(\Delta / \sigma)_{\max }=0.001$

$$
\Delta \rho_{\max }=0.49 \mathrm{e} \AA^{-3}
$$

$\Delta \rho_{\min }=-0.24$ e $\AA^{-3}$

\section{Special details}

Geometry. All e.s.d.'s (except the e.s.d. in the dihedral angle between two 1.s. planes) are estimated using the full covariance matrix. The cell e.s.d.'s are taken into account individually in the estimation of e.s.d.'s in distances, angles and torsion angles; correlations between e.s.d.'s in cell parameters are only used when they are defined by crystal symmetry. An approximate (isotropic) treatment of cell e.s.d.'s is used for estimating e.s.d.'s involving 1.s. planes.

Refinement. Refinement of $F^{2}$ against ALL reflections. The weighted $R$-factor $w R$ and goodness of fit $S$ are based on $F^{2}$, conventional $R$-factors $R$ are based on $F$, with $F$ set to zero for negative $F^{2}$. The threshold expression of $F^{2}>\sigma\left(F^{2}\right)$ is used only for calculating $R$-factors(gt) etc. and is not relevant to the choice of reflections for refinement. $R$-factors based on $F^{2}$ are statistically about twice as large as those based on $F$, and $R$ - factors based on ALL data will be even larger.

Fractional atomic coordinates and isotropic or equivalent isotropic displacement parameters $\left(\hat{A}^{2}\right)$

\begin{tabular}{lllll}
\hline & $x$ & $y$ & $z$ & $U_{\text {iso }} * / U_{\text {eq }}$ \\
\hline C1 & $0.19328(18)$ & $0.3613(2)$ & $0.06380(8)$ & $0.0188(4)$ \\
C2 & $0.22310(19)$ & $0.4340(2)$ & $0.01764(8)$ & $0.0209(4)$ \\
H2 & 0.1689 & 0.4962 & -0.0058 & $0.025^{*}$ \\
C3 & $0.35086(19)$ & $0.3998(2)$ & $0.01087(8)$ & $0.0213(4)$ \\
H3 & 0.3976 & 0.4334 & -0.0184 & $0.026^{*}$ \\
C4 & $0.39337(18)$ & $0.3108(2)$ & $0.05365(8)$ & $0.0191(4)$ \\
C5 & $0.08909(18)$ & $0.3614(2)$ & $0.16627(8)$ & $0.0205(4)$ \\
C6 & $0.0223(2)$ & $0.2789(3)$ & $0.20342(9)$ & $0.0247(5)$ \\
H6 & -0.0398 & 0.2060 & 0.1904 & $0.030^{*}$ \\
C7 & $0.0456(2)$ & $0.3020(3)$ & $0.25921(9)$ & $0.0270(5)$ \\
H7 & -0.0005 & 0.2448 & 0.2841 & $0.032^{*}$ \\
C8 & $0.1353(2)$ & $0.4078(3)$ & $0.27864(9)$ & $0.0270(5)$ \\
H8 & 0.1505 & 0.4242 & 0.3168 & $0.032^{*}$ \\
C9 & $0.2031(2)$ & $0.4898(3)$ & $0.24222(9)$ & $0.0284(5)$ \\
H9 & 0.2658 & 0.5617 & 0.2555 & $0.034^{*}$ \\
C10 & $0.18003(19)$ & $0.4675(3)$ & $0.18634(9)$ & $0.0249(5)$ \\
H10 & 0.2265 & 0.5249 & 0.1616 & $0.030^{*}$ \\
C11 & $0.01519(19)$ & $0.1311(2)$ & $0.08714(8)$ & $0.0202(4)$ \\
C12 & $0.09903(19)$ & $0.0193(3)$ & $0.10970(9)$ & $0.0234(5)$ \\
H12 & 0.1751 & 0.0516 & 0.1288 & $0.028^{*}$ \\
C13 & $0.0715(2)$ & $-0.1378(3)$ & $0.10433(9)$ & $0.0270(5)$ \\
H13 & 0.1291 & -0.2131 & 0.1197 & $0.032^{*}$ \\
C14 & $-0.0391(2)$ & $-0.1867(3)$ & $0.07683(9)$ & $0.0292(5)$ \\
H14 & -0.0577 & -0.2950 & 0.0736 & $0.035^{*}$ \\
C15 & $-0.1225(2)$ & $-0.0770(3)$ & $0.05413(9)$ & $0.0303(5)$ \\
H15 & -0.1984 & -0.1100 & 0.0351 & $0.036^{*}$ \\
C16 & $-0.0955(2)$ & $0.0810(3)$ & $0.05923(9)$ & $0.0248(5)$ \\
H16 & -0.1530 & 0.1558 & 0.0435 & $0.030^{*}$ \\
C17 & $0.5853(2)$ & $0.3097(2)$ & $0.14057(9)$ & $0.0252(5)$ \\
C18 & $0.5087(2)$ & $0.2928(3)$ & $0.18342(10)$ & $0.0378(6)$ \\
H18 & 0.4317 & 0.2381 & 0.1777 & $0.045^{*}$ \\
C19 & $0.5435(3)$ & $0.3546(4)$ & $0.23426(12)$ & $0.0595(9)$ \\
& & &
\end{tabular}




$\begin{array}{lllll}\mathrm{H} 19 & 0.4902 & 0.3431 & 0.2632 & 0.071^{*} \\ \mathrm{C} 20 & 0.6558(3) & 0.4332(4) & 0.24305(15) & 0.0698(12) \\ \mathrm{H} 20 & 0.6795 & 0.4757 & 0.2781 & 0.084^{*} \\ \mathrm{C} 21 & 0.7333(3) & 0.4501(3) & 0.20137(15) & 0.0592(10) \\ \mathrm{H} 21 & 0.8108 & 0.5034 & 0.2077 & 0.071^{*} \\ \mathrm{C} 22 & 0.6986(2) & 0.3893(3) & 0.15011(12) & 0.0390(6) \\ \mathrm{H} 22 & 0.7521 & 0.4019 & 0.1213 & 0.047^{*} \\ \mathrm{C} 23 & 0.51056(19) & 0.0292(2) & 0.08703(8) & 0.0205(4) \\ \mathrm{C} 24 & 0.4083(2) & -0.0493(3) & 0.06230(9) & 0.0264(5) \\ \mathrm{H} 24 & 0.3509 & 0.0047 & 0.0376 & 0.032^{*} \\ \mathrm{C} 25 & 0.3891(2) & -0.2063(3) & 0.07329(10) & 0.0316(5) \\ \mathrm{H} 25 & 0.3185 & -0.2588 & 0.0562 & 0.038^{*} \\ \mathrm{C} 26 & 0.4717(2) & -0.2864(3) & 0.10876(10) & 0.0349(6) \\ \mathrm{H} 26 & 0.4575 & -0.3932 & 0.1168 & 0.042^{*} \\ \mathrm{C} 27 & 0.5758(3) & -0.2098(3) & 0.13253(11) & 0.0405(6) \\ \mathrm{H} 27 & 0.6346 & -0.2650 & 0.1562 & 0.049^{*} \\ \mathrm{C} 28 & 0.5942(2) & -0.0540(3) & 0.12202(10) & 0.0315(5) \\ \mathrm{H} 28 & 0.6653 & -0.0022 & 0.1390 & 0.038^{*} \\ \mathrm{O} 1 & 0.29720(12) & 0.28470(16) & 0.08728(6) & 0.0202(3) \\ \mathrm{P} 1 & 0.04394(5) & 0.34210(6) & 0.09286(2) & 0.02035(15) \\ \mathrm{P} 2 & 0.54811(5) & 0.23216(6) & 0.07140(2) & 0.02108(15) \\ & & & & \end{array}$

Atomic displacement parameters $\left(\AA^{2}\right)$

\begin{tabular}{lllllll}
\hline & $U^{11}$ & $U^{22}$ & $U^{33}$ & $U^{12}$ & $U^{13}$ & $U^{23}$ \\
\hline C1 & $0.0193(10)$ & $0.0148(10)$ & $0.0221(10)$ & $0.0016(8)$ & $-0.0010(8)$ & $-0.0015(8)$ \\
C2 & $0.0247(11)$ & $0.0151(10)$ & $0.0223(11)$ & $-0.0027(8)$ & $-0.0027(8)$ & $0.0022(8)$ \\
C3 & $0.0237(10)$ & $0.0190(11)$ & $0.0213(11)$ & $-0.0057(8)$ & $0.0022(8)$ & $0.0015(8)$ \\
C4 & $0.0197(10)$ & $0.0165(10)$ & $0.0215(10)$ & $-0.0032(8)$ & $0.0041(8)$ & $-0.0015(8)$ \\
C5 & $0.0174(10)$ & $0.0204(11)$ & $0.0238(11)$ & $0.0051(8)$ & $0.0017(8)$ & $-0.0031(9)$ \\
C6 & $0.0223(11)$ & $0.0248(12)$ & $0.0270(11)$ & $-0.0023(9)$ & $0.0018(9)$ & $-0.0008(9)$ \\
C7 & $0.0258(11)$ & $0.0301(13)$ & $0.0257(12)$ & $0.0011(9)$ & $0.0054(9)$ & $0.0008(9)$ \\
C8 & $0.0251(11)$ & $0.0324(13)$ & $0.0233(11)$ & $0.0058(10)$ & $0.0002(9)$ & $-0.0058(9)$ \\
C9 & $0.0235(11)$ & $0.0268(12)$ & $0.0345(13)$ & $-0.0017(9)$ & $0.0006(9)$ & $-0.0091(10)$ \\
C10 & $0.0225(11)$ & $0.0214(11)$ & $0.0310(12)$ & $-0.0001(9)$ & $0.0039(9)$ & $-0.0013(9)$ \\
C11 & $0.0215(10)$ & $0.0213(11)$ & $0.0182(10)$ & $-0.0021(8)$ & $0.0046(8)$ & $-0.0014(8)$ \\
C12 & $0.0204(10)$ & $0.0246(12)$ & $0.0253(11)$ & $-0.0011(9)$ & $0.0016(8)$ & $0.0021(9)$ \\
C13 & $0.0304(12)$ & $0.0229(12)$ & $0.0284(12)$ & $0.0034(9)$ & $0.0058(9)$ & $0.0046(9)$ \\
C14 & $0.0380(13)$ & $0.0228(12)$ & $0.0274(12)$ & $-0.0071(10)$ & $0.0062(10)$ & $-0.0034(9)$ \\
C15 & $0.0304(12)$ & $0.0308(13)$ & $0.0288(12)$ & $-0.0080(10)$ & $-0.0026(10)$ & $-0.0057(10)$ \\
C16 & $0.0243(11)$ & $0.0274(12)$ & $0.0227(11)$ & $0.0000(9)$ & $0.0012(9)$ & $-0.0002(9)$ \\
C17 & $0.0244(11)$ & $0.0183(11)$ & $0.0318(12)$ & $0.0071(9)$ & $-0.0049(9)$ & $-0.0025(9)$ \\
C18 & $0.0348(13)$ & $0.0491(16)$ & $0.0288(13)$ & $0.0103(12)$ & $-0.0022(10)$ & $-0.0075(11)$ \\
C19 & $0.060(2)$ & $0.083(2)$ & $0.0337(15)$ & $0.0341(18)$ & $-0.0099(14)$ & $-0.0188(15)$ \\
C20 & $0.075(2)$ & $0.064(2)$ & $0.064(2)$ & $0.0447(19)$ & $-0.0437(19)$ & $-0.0425(18)$ \\
C21 & $0.0466(17)$ & $0.0303(15)$ & $0.094(3)$ & $0.0147(13)$ & $-0.0409(18)$ & $-0.0256(16)$ \\
C22 & $0.0279(12)$ & $0.0226(13)$ & $0.0642(18)$ & $0.0055(10)$ & $-0.0130(12)$ & $-0.0052(12)$ \\
C23 & $0.0235(10)$ & $0.0174(11)$ & $0.0215(11)$ & $0.0031(8)$ & $0.0076(8)$ & $-0.0015(8)$ \\
& & & & & &
\end{tabular}




\begin{tabular}{lllllll}
$\mathrm{C} 24$ & $0.0282(12)$ & $0.0214(12)$ & $0.0294(12)$ & $0.0034(9)$ & $0.0009(9)$ & $-0.0032(9)$ \\
$\mathrm{C} 25$ & $0.0338(13)$ & $0.0218(12)$ & $0.0403(14)$ & $-0.0028(10)$ & $0.0104(11)$ & $-0.0096(10)$ \\
$\mathrm{C} 26$ & $0.0528(16)$ & $0.0179(12)$ & $0.0360(13)$ & $0.0050(11)$ & $0.0173(12)$ & $0.0014(10)$ \\
C27 & $0.0542(16)$ & $0.0285(14)$ & $0.0375(14)$ & $0.0123(12)$ & $-0.0063(12)$ & $0.0038(11)$ \\
C28 & $0.0349(13)$ & $0.0259(12)$ & $0.0325(13)$ & $0.0035(10)$ & $-0.0055(10)$ & $0.0000(10)$ \\
O1 & $0.0189(7)$ & $0.0204(8)$ & $0.0214(7)$ & $0.0014(6)$ & $0.0039(6)$ & $0.0043(6)$ \\
P1 & $0.0184(3)$ & $0.0194(3)$ & $0.0232(3)$ & $0.0013(2)$ & $0.0013(2)$ & $0.0004(2)$ \\
P2 & $0.0185(3)$ & $0.0207(3)$ & $0.0244(3)$ & $0.0002(2)$ & $0.0041(2)$ & $0.0021(2)$ \\
\hline
\end{tabular}

Geometric parameters $\left(\AA,{ }^{\circ}\right)$

\begin{tabular}{|c|c|c|c|}
\hline $\mathrm{C} 1-\mathrm{C} 2$ & $1.352(3)$ & $\mathrm{C} 14-\mathrm{H} 14$ & 0.9500 \\
\hline $\mathrm{C} 1-\mathrm{O} 1$ & $1.381(2)$ & $\mathrm{C} 15-\mathrm{C} 16$ & $1.386(3)$ \\
\hline $\mathrm{C} 1-\mathrm{P} 1$ & $1.808(2)$ & C15-H15 & 0.9500 \\
\hline $\mathrm{C} 2-\mathrm{C} 3$ & $1.422(3)$ & $\mathrm{C} 16-\mathrm{H} 16$ & 0.9500 \\
\hline $\mathrm{C} 2-\mathrm{H} 2$ & 0.9500 & $\mathrm{C} 17-\mathrm{C} 18$ & $1.390(3)$ \\
\hline $\mathrm{C} 3-\mathrm{C} 4$ & $1.349(3)$ & $\mathrm{C} 17-\mathrm{C} 22$ & $1.396(3)$ \\
\hline $\mathrm{C} 3-\mathrm{H} 3$ & 0.9500 & $\mathrm{C} 17-\mathrm{P} 2$ & $1.839(2)$ \\
\hline $\mathrm{C} 4-\mathrm{O} 1$ & $1.386(2)$ & $\mathrm{C} 18-\mathrm{C} 19$ & $1.381(4)$ \\
\hline $\mathrm{C} 4-\mathrm{P} 2$ & $1.812(2)$ & C18-H18 & 0.9500 \\
\hline $\mathrm{C} 5-\mathrm{C} 6$ & $1.393(3)$ & $\mathrm{C} 19-\mathrm{C} 20$ & $1.382(5)$ \\
\hline $\mathrm{C} 5-\mathrm{C} 10$ & $1.395(3)$ & C19-H19 & 0.9500 \\
\hline $\mathrm{C} 5-\mathrm{P} 1$ & $1.838(2)$ & $\mathrm{C} 20-\mathrm{C} 21$ & $1.373(5)$ \\
\hline $\mathrm{C} 6-\mathrm{C} 7$ & $1.388(3)$ & $\mathrm{C} 20-\mathrm{H} 20$ & 0.9500 \\
\hline $\mathrm{C} 6-\mathrm{H} 6$ & 0.9500 & $\mathrm{C} 21-\mathrm{C} 22$ & $1.387(4)$ \\
\hline $\mathrm{C} 7-\mathrm{C} 8$ & $1.379(3)$ & $\mathrm{C} 21-\mathrm{H} 21$ & 0.9500 \\
\hline $\mathrm{C} 7-\mathrm{H} 7$ & 0.9500 & $\mathrm{C} 22-\mathrm{H} 22$ & 0.9500 \\
\hline $\mathrm{C} 8-\mathrm{C} 9$ & $1.385(3)$ & $\mathrm{C} 23-\mathrm{C} 24$ & $1.386(3)$ \\
\hline $\mathrm{C} 8-\mathrm{H} 8$ & 0.9500 & $\mathrm{C} 23-\mathrm{C} 28$ & $1.390(3)$ \\
\hline $\mathrm{C} 9-\mathrm{C} 10$ & $1.389(3)$ & $\mathrm{C} 23-\mathrm{P} 2$ & $1.830(2)$ \\
\hline C9-H9 & 0.9500 & $\mathrm{C} 24-\mathrm{C} 25$ & $1.388(3)$ \\
\hline $\mathrm{C} 10-\mathrm{H} 10$ & 0.9500 & $\mathrm{C} 24-\mathrm{H} 24$ & 0.9500 \\
\hline $\mathrm{C} 11-\mathrm{C} 16$ & $1.392(3)$ & $\mathrm{C} 25-\mathrm{C} 26$ & $1.377(4)$ \\
\hline $\mathrm{C} 11-\mathrm{C} 12$ & $1.398(3)$ & $\mathrm{C} 25-\mathrm{H} 25$ & 0.9500 \\
\hline $\mathrm{C} 11-\mathrm{P} 1$ & $1.835(2)$ & $\mathrm{C} 26-\mathrm{C} 27$ & $1.384(4)$ \\
\hline $\mathrm{C} 12-\mathrm{C} 13$ & $1.380(3)$ & $\mathrm{C} 26-\mathrm{H} 26$ & 0.9500 \\
\hline $\mathrm{C} 12-\mathrm{H} 12$ & 0.9500 & $\mathrm{C} 27-\mathrm{C} 28$ & $1.375(4)$ \\
\hline $\mathrm{C} 13-\mathrm{C} 14$ & $1.385(3)$ & $\mathrm{C} 27-\mathrm{H} 27$ & 0.9500 \\
\hline $\mathrm{C} 13-\mathrm{H} 13$ & 0.9500 & $\mathrm{C} 28-\mathrm{H} 28$ & 0.9500 \\
\hline $\mathrm{C} 14-\mathrm{C} 15$ & $1.384(3)$ & & \\
\hline $\mathrm{C} 2-\mathrm{C} 1-\mathrm{O} 1$ & $109.54(17)$ & $\mathrm{C} 15-\mathrm{C} 16-\mathrm{H} 16$ & 119.7 \\
\hline $\mathrm{C} 2-\mathrm{C} 1-\mathrm{P} 1$ & $130.13(16)$ & $\mathrm{C} 11-\mathrm{C} 16-\mathrm{H} 16$ & 119.7 \\
\hline $\mathrm{O} 1-\mathrm{C} 1-\mathrm{P} 1$ & $120.16(14)$ & $\mathrm{C} 18-\mathrm{C} 17-\mathrm{C} 22$ & $118.7(2)$ \\
\hline $\mathrm{C} 1-\mathrm{C} 2-\mathrm{C} 3$ & $107.06(18)$ & $\mathrm{C} 18-\mathrm{C} 17-\mathrm{P} 2$ & $124.21(18)$ \\
\hline $\mathrm{C} 1-\mathrm{C} 2-\mathrm{H} 2$ & 126.5 & $\mathrm{C} 22-\mathrm{C} 17-\mathrm{P} 2$ & 117.14 (19) \\
\hline $\mathrm{C} 3-\mathrm{C} 2-\mathrm{H} 2$ & 126.5 & $\mathrm{C} 19-\mathrm{C} 18-\mathrm{C} 17$ & $120.6(3)$ \\
\hline $\mathrm{C} 4-\mathrm{C} 3-\mathrm{C} 2$ & $107.29(18)$ & $\mathrm{C} 19-\mathrm{C} 18-\mathrm{H} 18$ & 119.7 \\
\hline
\end{tabular}




\begin{tabular}{|c|c|c|c|}
\hline $\mathrm{C} 4-\mathrm{C} 3-\mathrm{H} 3$ & 126.4 & $\mathrm{C} 17-\mathrm{C} 18-\mathrm{H} 18$ & 119.7 \\
\hline $\mathrm{C} 2-\mathrm{C} 3-\mathrm{H} 3$ & 126.4 & $\mathrm{C} 18-\mathrm{C} 19-\mathrm{C} 20$ & $120.0(3)$ \\
\hline $\mathrm{C} 3-\mathrm{C} 4-\mathrm{O} 1$ & $109.38(17)$ & $\mathrm{C} 18-\mathrm{C} 19-\mathrm{H} 19$ & 120.0 \\
\hline $\mathrm{C} 3-\mathrm{C} 4-\mathrm{P} 2$ & $130.27(16)$ & $\mathrm{C} 20-\mathrm{C} 19-\mathrm{H} 19$ & 120.0 \\
\hline $\mathrm{O} 1-\mathrm{C} 4-\mathrm{P} 2$ & $120.34(14)$ & $\mathrm{C} 21-\mathrm{C} 20-\mathrm{C} 19$ & $120.2(3)$ \\
\hline $\mathrm{C} 6-\mathrm{C} 5-\mathrm{C} 10$ & 118.57 (19) & $\mathrm{C} 21-\mathrm{C} 20-\mathrm{H} 20$ & 119.9 \\
\hline $\mathrm{C} 6-\mathrm{C} 5-\mathrm{P} 1$ & $119.10(16)$ & $\mathrm{C} 19-\mathrm{C} 20-\mathrm{H} 20$ & 119.9 \\
\hline $\mathrm{C} 10-\mathrm{C} 5-\mathrm{P} 1$ & $122.02(16)$ & $\mathrm{C} 20-\mathrm{C} 21-\mathrm{C} 22$ & $120.0(3)$ \\
\hline $\mathrm{C} 7-\mathrm{C} 6-\mathrm{C} 5$ & $120.7(2)$ & $\mathrm{C} 20-\mathrm{C} 21-\mathrm{H} 21$ & 120.0 \\
\hline $\mathrm{C} 7-\mathrm{C} 6-\mathrm{H} 6$ & 119.6 & $\mathrm{C} 22-\mathrm{C} 21-\mathrm{H} 21$ & 120.0 \\
\hline $\mathrm{C} 5-\mathrm{C} 6-\mathrm{H} 6$ & 119.6 & $\mathrm{C} 21-\mathrm{C} 22-\mathrm{C} 17$ & $120.4(3)$ \\
\hline $\mathrm{C} 8-\mathrm{C} 7-\mathrm{C} 6$ & $120.3(2)$ & $\mathrm{C} 21-\mathrm{C} 22-\mathrm{H} 22$ & 119.8 \\
\hline $\mathrm{C} 8-\mathrm{C} 7-\mathrm{H} 7$ & 119.9 & $\mathrm{C} 17-\mathrm{C} 22-\mathrm{H} 22$ & 119.8 \\
\hline $\mathrm{C} 6-\mathrm{C} 7-\mathrm{H} 7$ & 119.9 & $\mathrm{C} 24-\mathrm{C} 23-\mathrm{C} 28$ & $118.4(2)$ \\
\hline $\mathrm{C} 7-\mathrm{C} 8-\mathrm{C} 9$ & $119.7(2)$ & $\mathrm{C} 24-\mathrm{C} 23-\mathrm{P} 2$ & $123.17(16)$ \\
\hline $\mathrm{C} 7-\mathrm{C} 8-\mathrm{H} 8$ & 120.2 & $\mathrm{C} 28-\mathrm{C} 23-\mathrm{P} 2$ & $118.26(17)$ \\
\hline $\mathrm{C} 9-\mathrm{C} 8-\mathrm{H} 8$ & 120.2 & $\mathrm{C} 23-\mathrm{C} 24-\mathrm{C} 25$ & $120.5(2)$ \\
\hline $\mathrm{C} 8-\mathrm{C} 9-\mathrm{C} 10$ & $120.3(2)$ & $\mathrm{C} 23-\mathrm{C} 24-\mathrm{H} 24$ & 119.8 \\
\hline $\mathrm{C} 8-\mathrm{C} 9-\mathrm{H} 9$ & 119.8 & $\mathrm{C} 25-\mathrm{C} 24-\mathrm{H} 24$ & 119.8 \\
\hline $\mathrm{C} 10-\mathrm{C} 9-\mathrm{H} 9$ & 119.8 & $\mathrm{C} 26-\mathrm{C} 25-\mathrm{C} 24$ & $120.5(2)$ \\
\hline $\mathrm{C} 9-\mathrm{C} 10-\mathrm{C} 5$ & $120.4(2)$ & $\mathrm{C} 26-\mathrm{C} 25-\mathrm{H} 25$ & 119.8 \\
\hline $\mathrm{C} 9-\mathrm{C} 10-\mathrm{H} 10$ & 119.8 & $\mathrm{C} 24-\mathrm{C} 25-\mathrm{H} 25$ & 119.8 \\
\hline $\mathrm{C} 5-\mathrm{C} 10-\mathrm{H} 10$ & 119.8 & $\mathrm{C} 25-\mathrm{C} 26-\mathrm{C} 27$ & $119.3(2)$ \\
\hline $\mathrm{C} 16-\mathrm{C} 11-\mathrm{C} 12$ & $118.9(2)$ & $\mathrm{C} 25-\mathrm{C} 26-\mathrm{H} 26$ & 120.3 \\
\hline $\mathrm{C} 16-\mathrm{C} 11-\mathrm{P} 1$ & $118.21(16)$ & $\mathrm{C} 27-\mathrm{C} 26-\mathrm{H} 26$ & 120.3 \\
\hline $\mathrm{C} 12-\mathrm{C} 11-\mathrm{P} 1$ & $122.94(16)$ & $\mathrm{C} 28-\mathrm{C} 27-\mathrm{C} 26$ & $120.2(2)$ \\
\hline $\mathrm{C} 13-\mathrm{C} 12-\mathrm{C} 11$ & $120.2(2)$ & $\mathrm{C} 28-\mathrm{C} 27-\mathrm{H} 27$ & 119.9 \\
\hline $\mathrm{C} 13-\mathrm{C} 12-\mathrm{H} 12$ & 119.9 & $\mathrm{C} 26-\mathrm{C} 27-\mathrm{H} 27$ & 119.9 \\
\hline $\mathrm{C} 11-\mathrm{C} 12-\mathrm{H} 12$ & 119.9 & $\mathrm{C} 27-\mathrm{C} 28-\mathrm{C} 23$ & $121.1(2)$ \\
\hline $\mathrm{C} 12-\mathrm{C} 13-\mathrm{C} 14$ & $120.6(2)$ & $\mathrm{C} 27-\mathrm{C} 28-\mathrm{H} 28$ & 119.4 \\
\hline $\mathrm{C} 12-\mathrm{C} 13-\mathrm{H} 13$ & 119.7 & $\mathrm{C} 23-\mathrm{C} 28-\mathrm{H} 28$ & 119.4 \\
\hline $\mathrm{C} 14-\mathrm{C} 13-\mathrm{H} 13$ & 119.7 & $\mathrm{C} 1-\mathrm{O} 1-\mathrm{C} 4$ & $106.71(15)$ \\
\hline $\mathrm{C} 15-\mathrm{C} 14-\mathrm{C} 13$ & $119.7(2)$ & $\mathrm{C} 1-\mathrm{P} 1-\mathrm{C} 11$ & $101.99(9)$ \\
\hline $\mathrm{C} 15-\mathrm{C} 14-\mathrm{H} 14$ & 120.1 & $\mathrm{C} 1-\mathrm{P} 1-\mathrm{C} 5$ & $101.71(9)$ \\
\hline $\mathrm{C} 13-\mathrm{C} 14-\mathrm{H} 14$ & 120.1 & $\mathrm{C} 11-\mathrm{P} 1-\mathrm{C} 5$ & $101.16(9)$ \\
\hline $\mathrm{C} 14-\mathrm{C} 15-\mathrm{C} 16$ & $120.0(2)$ & $\mathrm{C} 4-\mathrm{P} 2-\mathrm{C} 23$ & $101.00(9)$ \\
\hline $\mathrm{C} 14-\mathrm{C} 15-\mathrm{H} 15$ & 120.0 & $\mathrm{C} 4-\mathrm{P} 2-\mathrm{C} 17$ & $102.65(9)$ \\
\hline $\mathrm{C} 16-\mathrm{C} 15-\mathrm{H} 15$ & 120.0 & $\mathrm{C} 23-\mathrm{P} 2-\mathrm{C} 17$ & $100.63(10)$ \\
\hline $\mathrm{C} 15-\mathrm{C} 16-\mathrm{C} 11$ & $120.6(2)$ & & \\
\hline $\mathrm{O} 1-\mathrm{C} 1-\mathrm{C} 2-\mathrm{C} 3$ & $1.1(2)$ & $\mathrm{C} 25-\mathrm{C} 26-\mathrm{C} 27-\mathrm{C} 28$ & $1.9(4)$ \\
\hline $\mathrm{P} 1-\mathrm{C} 1-\mathrm{C} 2-\mathrm{C} 3$ & $-173.96(16)$ & $\mathrm{C} 26-\mathrm{C} 27-\mathrm{C} 28-\mathrm{C} 23$ & $-0.9(4)$ \\
\hline $\mathrm{C} 1-\mathrm{C} 2-\mathrm{C} 3-\mathrm{C} 4$ & $-1.2(2)$ & $\mathrm{C} 24-\mathrm{C} 23-\mathrm{C} 28-\mathrm{C} 27$ & $-0.7(3)$ \\
\hline $\mathrm{C} 2-\mathrm{C} 3-\mathrm{C} 4-\mathrm{O} 1$ & $0.8(2)$ & $\mathrm{P} 2-\mathrm{C} 23-\mathrm{C} 28-\mathrm{C} 27$ & $-175.58(19)$ \\
\hline $\mathrm{C} 2-\mathrm{C} 3-\mathrm{C} 4-\mathrm{P} 2$ & $-177.79(16)$ & $\mathrm{C} 2-\mathrm{C} 1-\mathrm{O} 1-\mathrm{C} 4$ & $-0.7(2)$ \\
\hline $\mathrm{C} 10-\mathrm{C} 5-\mathrm{C} 6-\mathrm{C} 7$ & $0.1(3)$ & $\mathrm{P} 1-\mathrm{C} 1-\mathrm{O} 1-\mathrm{C} 4$ & $175.01(14)$ \\
\hline $\mathrm{P} 1-\mathrm{C} 5-\mathrm{C} 6-\mathrm{C} 7$ & $-173.69(16)$ & $\mathrm{C} 3-\mathrm{C} 4-\mathrm{O} 1-\mathrm{C} 1$ & $-0.1(2)$ \\
\hline $\mathrm{C} 5-\mathrm{C} 6-\mathrm{C} 7-\mathrm{C} 8$ & $0.2(3)$ & $\mathrm{P} 2-\mathrm{C} 4-\mathrm{O} 1-\mathrm{C} 1$ & $178.63(14)$ \\
\hline
\end{tabular}




$\begin{array}{llll}\mathrm{C} 6-\mathrm{C} 7-\mathrm{C} 8-\mathrm{C} 9 & -0.6(3) & \mathrm{C} 2-\mathrm{C} 1-\mathrm{P} 1-\mathrm{C} 11 & 114.0(2) \\ \mathrm{C} 7-\mathrm{C} 8-\mathrm{C} 9-\mathrm{C} 10 & 0.8(3) & \mathrm{O} 1-\mathrm{C} 1-\mathrm{P} 1-\mathrm{C} 11 & -60.70(17) \\ \mathrm{C} 8-\mathrm{C} 9-\mathrm{C} 10-\mathrm{C} 5 & -0.5(3) & \mathrm{C} 2-\mathrm{C} 1-\mathrm{P} 1-\mathrm{C} 5 & -141.8(2) \\ \mathrm{C} 6-\mathrm{C} 5-\mathrm{C} 10-\mathrm{C} 9 & 0.1(3) & \mathrm{O} 1-\mathrm{C} 1-\mathrm{P} 1-\mathrm{C} 5 & 43.55(17) \\ \mathrm{P} 1-\mathrm{C} 5-\mathrm{C} 10-\mathrm{C} 9 & 173.68(16) & \mathrm{C} 16-\mathrm{C} 11-\mathrm{P} 1-\mathrm{C} 1 & -124.03(16) \\ \mathrm{C} 16-\mathrm{C} 11-\mathrm{C} 12-\mathrm{C} 13 & -0.2(3) & \mathrm{C} 12-\mathrm{C} 11-\mathrm{P} 1-\mathrm{C} 1 & 56.25(19) \\ \mathrm{P} 1-\mathrm{C} 11-\mathrm{C} 12-\mathrm{C} 13 & 179.52(16) & \mathrm{C} 16-\mathrm{C} 11-\mathrm{P} 1-\mathrm{C} 5 & 131.29(16) \\ \mathrm{C} 11-\mathrm{C} 12-\mathrm{C} 13-\mathrm{C} 14 & -0.2(3) & \mathrm{C} 12-\mathrm{C} 11-\mathrm{P} 1-\mathrm{C} 5 & -48.43(18) \\ \mathrm{C} 12-\mathrm{C} 13-\mathrm{C} 14-\mathrm{C} 15 & 0.5(3) & \mathrm{C} 6-\mathrm{C} 5-\mathrm{P} 1-\mathrm{C} 1 & -147.20(17) \\ \mathrm{C} 13-\mathrm{C} 14-\mathrm{C} 15-\mathrm{C} 16 & -0.4(3) & \mathrm{C} 10-\mathrm{C} 5-\mathrm{P} 1-\mathrm{C} 1 & 39.21(19) \\ \mathrm{C} 14-\mathrm{C} 15-\mathrm{C} 16-\mathrm{C} 11 & -0.1(3) & \mathrm{C} 6-\mathrm{C} 5-\mathrm{P} 1-\mathrm{C} 11 & -42.30(18) \\ \mathrm{C} 12-\mathrm{C} 11-\mathrm{C} 16-\mathrm{C} 15 & 0.4(3) & \mathrm{C} 10-\mathrm{C} 5-\mathrm{P} 1-\mathrm{C} 11 & 144.11(17) \\ \mathrm{P} 1-\mathrm{C} 11-\mathrm{C} 16-\mathrm{C} 15 & -179.37(17) & \mathrm{C} 3-\mathrm{C} 4-\mathrm{P} 2-\mathrm{C} 23 & -132.8(2) \\ \mathrm{C} 22-\mathrm{C} 17-\mathrm{C} 18-\mathrm{C} 19 & 0.6(4) & \mathrm{O} 1-\mathrm{C} 4-\mathrm{P} 2-\mathrm{C} 23 & 48.70(17) \\ \mathrm{P} 2-\mathrm{C} 17-\mathrm{C} 18-\mathrm{C} 19 & -180.0(2) & \mathrm{C} 3-\mathrm{C} 4-\mathrm{P} 2-\mathrm{C} 17 & 123.5(2) \\ \mathrm{C} 17-\mathrm{C} 18-\mathrm{C} 19-\mathrm{C} 20 & -0.5(4) & \mathrm{O} 1-\mathrm{C} 4-\mathrm{P} 2-\mathrm{C} 17 & -54.96(17) \\ \mathrm{C} 18-\mathrm{C} 19-\mathrm{C} 20-\mathrm{C} 21 & -0.1(5) & \mathrm{C} 24-\mathrm{C} 23-\mathrm{P} 2-\mathrm{C} 4 & 30.32(19) \\ \mathrm{C} 19-\mathrm{C} 20-\mathrm{C} 21-\mathrm{C} 22 & 0.5(4) & \mathrm{C} 28-\mathrm{C} 23-\mathrm{P} 2-\mathrm{C} 4 & -155.10(17) \\ \mathrm{C} 20-\mathrm{C} 21-\mathrm{C} 22-\mathrm{C} 17 & -0.5(4) & \mathrm{C} 24-\mathrm{C} 23-\mathrm{P} 2-\mathrm{C} 17 & 135.60(18) \\ \mathrm{C} 18-\mathrm{C} 17-\mathrm{C} 22-\mathrm{C} 21 & -0.1(3) & \mathrm{C} 28-\mathrm{C} 23-\mathrm{P} 2-\mathrm{C} 17 & -49.82(18) \\ \mathrm{P} 2-\mathrm{C} 17-\mathrm{C} 22-\mathrm{C} 21 & -179.55(19) & \mathrm{C} 18-\mathrm{C} 17-\mathrm{P} 2-\mathrm{C} 4 & 53.1(2) \\ \mathrm{C} 28-\mathrm{C} 23-\mathrm{C} 24-\mathrm{C} 25 & 1.3(3) & \mathrm{C} 22-\mathrm{C} 17-\mathrm{P} 2-\mathrm{C} 4 & -127.44(18) \\ \mathrm{P} 2-\mathrm{C} 23-\mathrm{C} 24-\mathrm{C} 25 & 175.88(16) & \mathrm{C} 18-\mathrm{C} 17-\mathrm{P} 2-\mathrm{C} 23 & -50.9(2) \\ \mathrm{C} 23-\mathrm{C} 24-\mathrm{C} 25-\mathrm{C} 26 & -0.3(3) & \mathrm{C} 22-\mathrm{C} 17-\mathrm{P} 2-\mathrm{C} 23 & 128.61(18) \\ \mathrm{C} 24-\mathrm{C} 25-\mathrm{C} 26-\mathrm{C} 27 & -1.4(3) & & \\ & & & \end{array}$

Hydrogen-bond geometry $\left(A,{ }^{\circ}\right)$

$\mathrm{Cg}$ is the centroid of ring $\mathrm{C} 17-\mathrm{C} 22$.

\begin{tabular}{lllll}
\hline$D-\mathrm{H} \cdots A$ & $D-\mathrm{H}$ & $\mathrm{H} \cdots A$ & $D \cdots A$ & $D-\mathrm{H} \cdots A$ \\
\hline $\mathrm{C} 27-\mathrm{H} 27 \cdots C g^{\mathrm{i}}$ & 0.95 & 3.11 & $3.736(3)$ & 125 \\
\hline
\end{tabular}

Symmetry code: (i) $x, y-1, z$. 